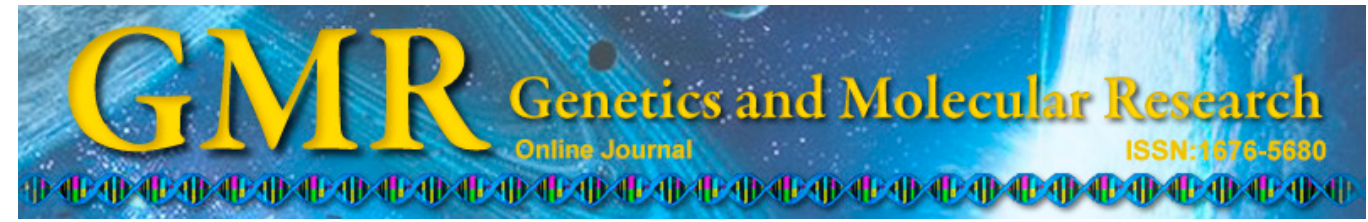

\title{
Interethnic variation of the $M M P-9$ microsatellite in Amerindian and Mexican Mestizo populations: considerations for genetic association studies
}

R. Camacho-Mejorado' ${ }^{1}$ G. Noris ${ }^{2}$, C. Santana ${ }^{2}$, J.J. Magaña ${ }^{3}$, A. Majluf-Cruz ${ }^{4}$, J. Arellano-Galindo ${ }^{5}$, A. De la Peña ${ }^{6,7}$, J. Hernández-Juárez ${ }^{4}$, E.S. Calderón-Aranda ${ }^{1}$,M.A. Meraz-Ríos ${ }^{8}$ and R. Gómez ${ }^{1}$

${ }^{1}$ Departament of Toxicology, Centre of Research and Advanced Studies of National Polytechnic Institute (Cinvestav-IPN), Mexico City, Mexico ${ }^{2}$ BIMODI Laboratory (Molecular Biology Diagnostic), Querétaro, México ${ }^{3}$ Laboratory of Genomic Medicine, Department of Genetics, National Rehabilitation Institute, Mexico City, Mexico

${ }^{4}$ Thrombosis, Haemostasis and Atherogenesis Medical Research Unit of the Mexican Institute of Social Security, Mexico City, Mexico ${ }^{5}$ Laboratory of Virology, Children Hospital of Mexico Federico Gómez, Mexico City, Mexico ${ }^{6}$ Departament of Pharmacology, Faculty of Medicine, National University of Mexico, Mexico City, Mexico ${ }^{7}$ Thrombosis and Fribrinolysis Laboratory, National Institute of Cardiology Ignacio Chávez, Mexico City, Mexico ${ }^{8}$ Departament of Molecular Biomedicine, Centre of Research and Advanced Studies of National Polytechnic Institute (Cinvestav-IPN), Mexico City, Mexico

Corresponding author: R. Gómez

E-mail:mrgomez@cinvestav.mx

Genet. Mol. Res. 14 (1): 2929-2939 (2015)

Received June 30, 2014

Accepted October 21, 2014

Published March 31, 2015

DOI http://dx.doi.org/10.4238/2015.March.31.24

ABSTRACT. We studied the interethnic variation of the $M M P$ 9 microsatellite in the Mestizo and Amerindian populations using 
blood samples collected from 435 healthy unrelated individuals from the Central Valley of Mexico. DNA samples were genotyped using the $-90(\mathrm{CA})_{12-27}$ repeat near the MMP transcriptional start site using capillary electrophoresis. Our data were compared with those from African, Asian, and European populations $(\mathrm{N}=729)$. Both Mestizo and Amerindian populations were in Hardy-Weinberg equilibrium $(\mathrm{P} \geq 0.05)$. However, strong genetic heterogeneity was found within the Mestizo population $(94 \%, \mathrm{P} \leq 0.0001)$, which exhibited the highest frequency of Amerindian, African, and European alleles. Likewise, Amerindians showed $6.7 \%$ variation among populations $(\mathrm{P} \leq 0.0001)$, suggesting a genetic substructure potentially associated with linguistic affiliations. These findings were corroborated with principal component and population differentiation analyses, which showed relative proximity among the Mestizos and their historical parental populations: Asian $\left(F_{\mathrm{ST}}\right.$ $\geq 0.05)$, European $\left(F_{\mathrm{ST}} \geq 0.09\right)$, and African $\left(F_{\mathrm{ST}} \geq 0.02\right)$. Nevertheless, important differences were found between Mestizo and Nahuas ( $\mathrm{P}$ $\leq 0.0001)$, and between Mestizo and Me'Phaas $(\mathrm{P} \leq 0.0001)$. These findings highlight the importance of determining local-specific patterns to establish the population variability of $M M P-9$ and other polymorphic markers. Validation of candidate markers is critical to identifying risk factors; however, this depends on knowledge of population genetic variation, which increases the possibility of finding true causative variants. We also show that dissimilar ethnic backgrounds might lead to spurious associations. Our study provides useful considerations for greater accuracy and robustness in future genetic association studies.

Key words: MMP-9; Polymorphisms; Population genetics; Ethnicity; Mexican Mestizo population; Amerindian

\section{INTRODUCTION}

Matrix metalloproteinase $9(M M P-9)$ is a zinc-dependent protein whose activity is primarily regulated by gene transcription (Van den Steen et al., 2002; Dabek et al., 2013). The $M M P-9$ gene contains a microsatellite sequence $\left[-90(\mathrm{CA})_{12-27}\right]$ close to the transcriptional start site, which modulates the promoter activity (Ferrand et al., 2002; Fornoni et al., 2002). Microsatellites (short tandem repeats, STRs), exert regulatory roles such as modification of gene expression patterns and transcription regulation that may influence protein function (Sawaya et al., 2013). Studies on the $M M P-9$ gene have associated the length of the dinucleotide repeat with promoter activity, suggesting that (CA) ${ }_{14}$ may cause a significant decrease (up to 50\%) in transcriptional activity in comparison to (CA $)_{21}$ (Shimajiri et al., 1999; Ferrand et al., 2002). Therefore, polymorphisms of the $M M P-9$ gene have been implicated in the susceptibility to various diseases such as cancer (Kader et al., 2006), lipid levels (Mazzotti et al., 2014), cardiovascular diseases (Fiotti et al., 2006), and Alzheimer's disease (Flex et al., 2013), among others.

Evaluation of the allelic distribution among different populations suggests interethnic differences in genetic variants of $M M P-9$ (Lacchini et al., 2010). In this regard, different reports have shown that several populations such as European, African-American, and Brazilian 
populations present a bimodal distribution (Ferrand et al., 2002; Lamblin et al., 2002; Lacchini et al., 2010). Meanwhile, Asian populations (Japan and Korea) exhibit a unimodal distribution (Maeda et al., 2001; Lee et al., 2010). In addition, the alleles (CA) 14 and (CA) $)_{21}$ exhibit higher incidence in populations from Europe (55\%) and Asia (45-60\%) (Van den Steen et al., 2002). In regard to American populations, previous studies in Brazilian populations showed that Afro descent (Salvador, Bahia) show high frequencies of $(\mathrm{CA})_{>_{21}}(62 \%)$ in comparison to European descent (Ribeirão Preto, São Paulo) $(49 \%)(\mathrm{P} \leq 0.002)$ (Lacchini et al., 2010). These interethnic differences may lead to spurious associations in terms of disease risk as well as drug response (Yasuda et al., 2008). Hence, genetic ancestry could act as a confounding factor in population stratification leading to a lack of reproducibility in genetic association studies among populations (Freedman et al., 2004; Choudhry et al., 2006).

Mexican Mestizos are an admixture population that emerged 520 years ago with the miscegenation among Amerindian, European, and African populations (Salazar-Flores et al., 2010). The Mestizo group represents $98 \%$ of the population, whereas the remaining $2 \%$ is represented by almost 60 different Amerindian groups (INEGI, 2007; CDI, 2010). Besides migrations, demographic events such as bottlenecks, founder effects, local drift, and rapid population growth have induced significant differences in allele frequencies across the country (Juarez-Cedillo et al., 2008; Noris et al., 2012). Consequently, heterogeneous genetic patterns have been produced. This genetic heterogeneity is mainly located in the central region of the country (Central Valley of Mexico, CVM), where contemporary migrations have resulted in a gene pool that could represent the complex genetic structure of the entire Mexican population (Noris et al., 2012).

Thus, the main aims of the present study were: 1) to assess the genetic variability of $M M P$ $9-90(\mathrm{CA})_{12-27}$ in the Mexican Mestizo and some Amerindian (Nahuas, Mazahuas, and Me'Phaas) populations; 2) to determine the interethnic variation within Mexican populations; and 3) to compare our results with previous data for other world populations. The knowledge of genetic diversity throughout the country could avoid spurious associations in future genetic studies.

\section{MATERIAL AND METHODS}

\section{Study populations}

Blood samples were collected from 435 unrelated individuals belonging to the Mestizo and Amerindian populations. The Mestizo population consisted of 300 healthy individuals (150 men and 150 women) with at least three generations of ancestors born in Mexico. This population was recruited from the states of Queretaro, Guanajuato, and Puebla (CVM). In addition, blood samples of 135 healthy individuals (41 men and 94 women) belonging to different Amerindian groups were collected from different geographic areas: Mazahuas $(\mathrm{N}=29)$ from Santa Rosa and Pueblo Nuevo, State of Mexico; Nahuas $(\mathrm{N}=71)$ from Cacaloc, Zoquitlán, Puebla; and Me'Phaas $(\mathrm{N}=35)$ from the uplands of Guerrero's Sierra, Guerrero (Figure 1).

Each individual signed an informed consent form validated by the Ethics Committee of the Bimodi Research Unit and the Thrombosis, Hemostasis and Atherogenesis Medical Research Unit (Unidad de Investigación Médica en Trombosis, Hemostasia y Aterogénesis) of the Mexican Institute of Social Security in Mexico City. Genealogical data were also obtained from each individual to ensure that the individuals were unrelated through at least three generations. 


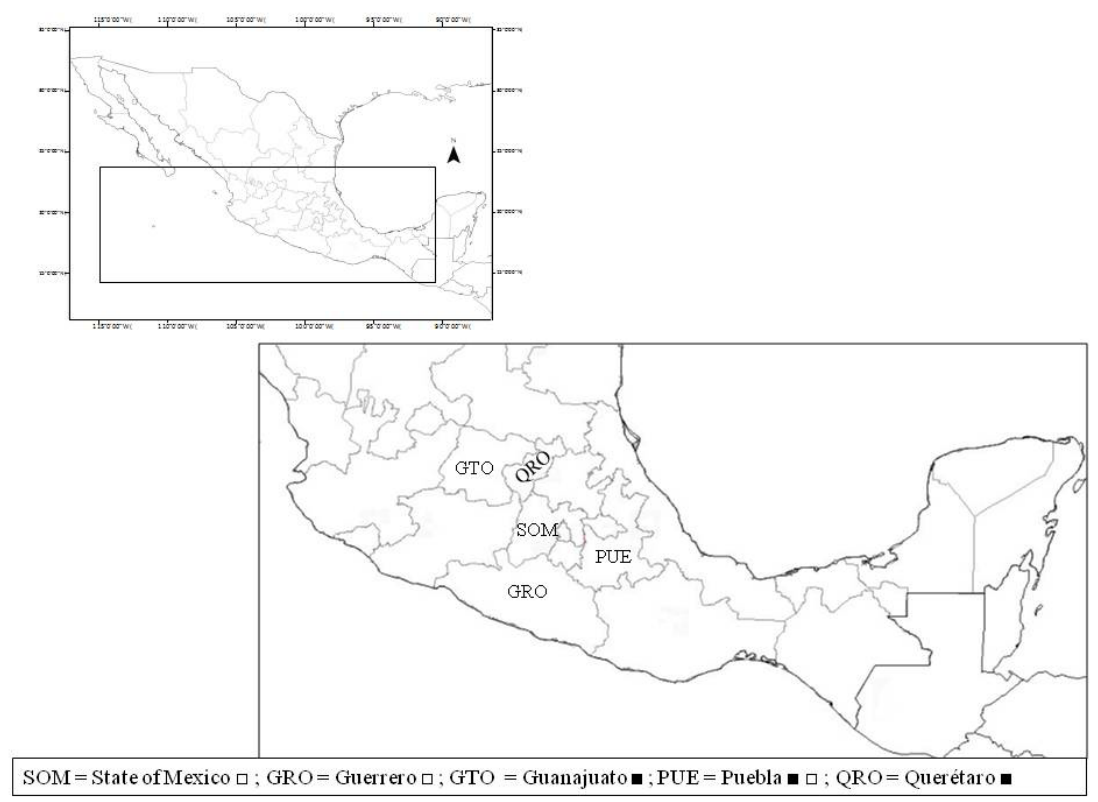

Figure 1. Geographical localization of sampling states. Filled squares: Mexican Mestizo population from Mexico, central region (GTO, PUE, QRO); Open squares: Amerindian populations: Tlapanecos (GRO), Mazahuas (SOM), Nahuas (PUE).

\section{Molecular analysis}

Genomic DNA was extracted from peripheral blood leukocytes using a Qiamp DNA Mini Kit (Qiagen; Düsseldorf, Germany). Polymerase chain reaction (PCR) amplification was performed with oligonucleotide primers to amplify a polymorphic $(\mathrm{CA})_{\mathrm{n}}$ repeat in the human $M M P-9$ gene as described previously (Fiotti et al., 2005). Approximately 10 ng target DNA was amplified using a Thermocycler Kyratec Supercycler SC200 (Queensland, Australia). The reaction was standardized in a $6 \mu \mathrm{L}$ total volume, containing $0.015 \mu \mathrm{M}$ primers, $1 \mathrm{X}$ Reaction Buffer with $\mathrm{NH}_{4} \mathrm{SO}_{2}, 2 \mathrm{mM} \mathrm{MgCl}, 200 \mu \mathrm{M}$ of each nucleotide (Thermo Fisher Scientific, Suwanee, GA, USA), 1 M betaine (Sigma-Aldrich, St. Louis, MO, USA), and 1 U Taq DNA polymerase (Thermo Fisher Scientific). The thermocycling procedure consisted of 35 cycles of denaturation at $94^{\circ} \mathrm{C}$ for $1 \mathrm{~min}$, annealing at $63^{\circ} \mathrm{C}$ for $1 \mathrm{~min}$, and extension at $72^{\circ} \mathrm{C}$ for $1 \mathrm{~min}$, followed by a final extension step of $10 \mathrm{~min}$ at $72^{\circ} \mathrm{C}$. The resulting amplicons were analyzed by capillary electrophoresis on the ABI Prism 3130XL Genetic Analyzer using the GeneMapper ID v.3.2. software (Applied Biosystems, Carlsbad, CA, USA).

\section{Sequence analysis}

DNA sequencing was performed to confirm the size of the alleles detected during our study using the ABI Big Dye Terminator Cycle Sequencing Ready Reaction kit (Applied Biosystems). PCR-amplified fragments were purified using BigDye XTerminator ${ }^{\circledR}$ Purification kit (Applied Biosystems) and visualized in an ABI PRISM 3130XL analyzer using the Sequencing Analysis v.5.3.1 software (Applied Biosystems). 


\section{Statistical analysis}

\section{Allele and genotype frequencies}

Allele and genotype frequencies, number of alleles $(k)$, and expected heterozygosity were estimated using the Arlequin v 3.5 software (Excoffier et al., 2005). Hardy-Weinberg expectation (HWE) was obtained by applying Weir and Cockerham's $F$ statistics, using the GENETIX v. 4.05.2 software with 10,000 permutations (Belkhir et al., 1996).

\section{Comparison with other populations}

To compare our data with those of other populations, genotype information was collected from previous reports (Table 1). A total of five populations $(\mathrm{N}=729)$ were included in the database and used for further analysis. These populations included individuals from France and England (Zhang et al., 2001; Lamblin et al., 2002), Japan and Korea (Maeda et al., 2001; Lee et al., 2010), and the African-American population from the USA (Ferrand et al., 2002). Population subdivision was assessed by analysis of molecular variance (AMOVA) using ethnicity as subdivision criteria with the Arlequin Software v.3.5. In addition, population differentiation and factorial correspondence analyses were conducted using the Arlequin Software v.3.5 (Excoffier et al., 2005) and the GENETIX v. 4.05.2 software (Belkhir et al., 1996), respectively.

\begin{tabular}{|c|c|c|c|}
\hline Population & Sample size & Region & Reference \\
\hline Mexican Mestizo & 300 & Central Mexico, Mexico & This study \\
\hline Nahua & 71 & Cacaloc, Zoquitlán, Puebla, Mexico & This study \\
\hline Mazahua & 29 & Santa Rosa, State of Mexico, Mexico & This study \\
\hline Tlapaneco & 35 & Uplands of Guerrero's Sierra, Guerrero, Mexico & This study \\
\hline British & 94 & Southampton, England & Zhang et al., 2001 \\
\hline Korean & 122 & Seoul, Korea & Lee et al., 2010 \\
\hline French & 226 & Lille, France & Lamblin et al., 2002 \\
\hline Japanese & 72 & Shiga, Japan & Maeda et al., 2001 \\
\hline African American & 215 & Philadelphia, USA & Ferrand et al., 2002 \\
\hline
\end{tabular}

\section{RESULTS}

\section{Statistical genetics}

Allele frequency distributions for the $-90(\mathrm{CA})_{12-27} M M P-9$ locus in Mestizo and Amerindian populations are shown in Table 2. The Mexican Mestizo population presented a bimodal distribution with 13 different alleles $(k=13)$, where MMP9-14 (0.22), MMP9-21 (0.30), MMP9-22 (0.17), and MMP9-23 (0.20) were the most frequent. These values correlated with the allele distribution reported in other populations (Maeda et al., 2001; Ferrand et al., 2002; Lacchini et al., 2010; Lee et al., 2010). The most frequent genotypes were: 14/21 (12.7\%), 21/21 (10.3\%), and 14/22 (8.3\%). Alternatively, the Amerindian populations showed a unimodal distribution. The Nahua population exhibited nine different alleles, with MMP9$22(0.40)$ the most frequent. The Mazahua and Tlapaneco populations exhibited five different alleles each; the most frequent were MMP9-21 (0.40) and MMP9-23 (0.36) in Mazahuas, and MMP9-23 (0.53) in Me'Phaas. 
Table 2. Allele frequency and descriptive parameters of the $-90(\mathrm{CA})_{\mathrm{n}} M M P-9$ microsatellite in the Mexican Mestizo, Nahua, Mazahua, and Tlapaneco populations.

\begin{tabular}{|c|c|c|c|c|}
\hline & \multicolumn{4}{|c|}{ Population } \\
\hline & Mexican Mestizo & Nahua & Mazahua & Tlapaneco \\
\hline Alleles $(\mathrm{N})$ & 600 & 142 & 58 & 70 \\
\hline 12 & 0.002 & - & - & - \\
\hline 14 & 0.223 & 0.056 & 0.103 & - \\
\hline 15 & 0.022 & 0.021 & - & - \\
\hline 17 & 0.003 & - & - & - \\
\hline 18 & 0.002 & - & - & - \\
\hline 19 & 0.022 & 0.021 & 0.017 & 0.014 \\
\hline 20 & 0.020 & 0.014 & - & - \\
\hline 21 & 0.297 & 0.218 & 0.397 & 0.157 \\
\hline 22 & 0.173 & 0.394 & 0.121 & 0.286 \\
\hline 23 & 0.198 & 0.232 & 0.362 & 0.529 \\
\hline 24 & 0.035 & 0.035 & - & 0.014 \\
\hline 25 & 0.002 & 0.007 & - & - \\
\hline \multirow[t]{2}{*}{27} & 0.002 & - & - & - \\
\hline & \multicolumn{4}{|c|}{ Descriptive statistics } \\
\hline$k$ & 13 & 9 & 5 & 5 \\
\hline$F_{\text {IS }}$ & 0.0229 & -0.0245 & 0.0625 & -0.0094 \\
\hline HWE (P) & 0.232 & 0.695 & 0.368 & 0.598 \\
\hline$H_{\mathrm{O}}$ & 0.77333 & 0.76056 & 0.65517 & 0.62857 \\
\hline$H_{\mathrm{E}}$ & 0.79146 & 0.74248 & 0.69812 & 0.62277 \\
\hline
\end{tabular}

Bold numbers show two important sets of microsatellite repeats (allele 14 and alleles 21-23) in which there is large genetic variability throughout the populations. $k=$ Number of found alleles; HWE $=$ Hardy Weinberg equilibrium, P value; Weir and Cockerham $F_{\mathrm{IS}} ; H_{\mathrm{O}}=$ observed heterozygosity; $H_{\mathrm{E}}=$ expected heterozygosity.

The genotype frequencies calculated in the different populations were as follows: Mazahuas were represented by the genotypes 21/23 (24\%) and 21/21 (21\%), whereas Nahuas showed a more homogeneous distribution characterized by the genotypes $21 / 22(17 \%), 22 / 22$ and $22 / 23(15 \%$, each), and $21 / 23(14 \%)$. On the other hand, the Tlapaneco population was mainly represented by the genotypes: 22/23 (40\%), 23/23 (23\%), and 21/23 (17\%).

Mestizo and Amerindian populations were in $\operatorname{HWE}(\mathrm{P} \geq 0.05)$ (Table 2).

The majority of alleles were sequenced to check the repeat length and verify allele assignation (data not shown).

\section{AMOVA}

To assess the heterogeneity between Mestizo and Amerindian populations, AMOVA test was performed. The results showed $5.96 \%$ heterogeneity between populations $(\mathrm{P} \leq 0.0001)$, whereas the highest variation was found within populations (94\%). In light of this evidence, AMOVA test was also performed among Amerindian populations (Mazahua, Nahua, and Tlapaneco). The results demonstrated 6.7\% variation among populations $(\mathrm{P} \leq 0.0001)$, whereas the highest variation (93\%) was found within populations. In addition, AMOVA was carried out using language as the subdivision criterion: Uto-Aztecan (Nahuas) and Oto-Manguean (Mazahuas and Me'Phaas). The results showed $5.03 \%$ heterogeneity among populations and $94.97 \%$ variation within populations $(\mathrm{P} \leq 0.0001)$, suggesting a genetic substructure that might possibly be associated with linguistic affiliations.

In an attempt to determine the genetic differences among populations, principal com- 
ponent analyses (PCA) were performed. The first component separated the Mazahua and Tlapaneco populations from the Mestizos and Nahuas, and the second component set apart the Mestizo and Mazahua populations from the Nahuas and Me'Phaas (Figure 2A).

A population differentiation analysis was performed to detect genetic differences among the Mestizo and Amerindian populations. In this regard, important differences were found between the Mestizo and Nahuas ( $\mathrm{P} \leq 0.0001)$, as well as between the Mestizo and Me'Phaas (0.0001). In contrast, Mestizos and Mazahuas showed no significant differences $(0.6570 \pm 0.0427)$. Interestingly, even among Amerindian populations important differences were found, in which the Nahuas exhibited significant differences with the Mazahuas $(0.0570$ $\pm 0.0181)$ and Me'Phaas $(0.0084 \pm 0.0033)$. In addition, the Mazahuas showed statistical differences with the Me'Phaas $(0.0048 \pm 0.0022)$.
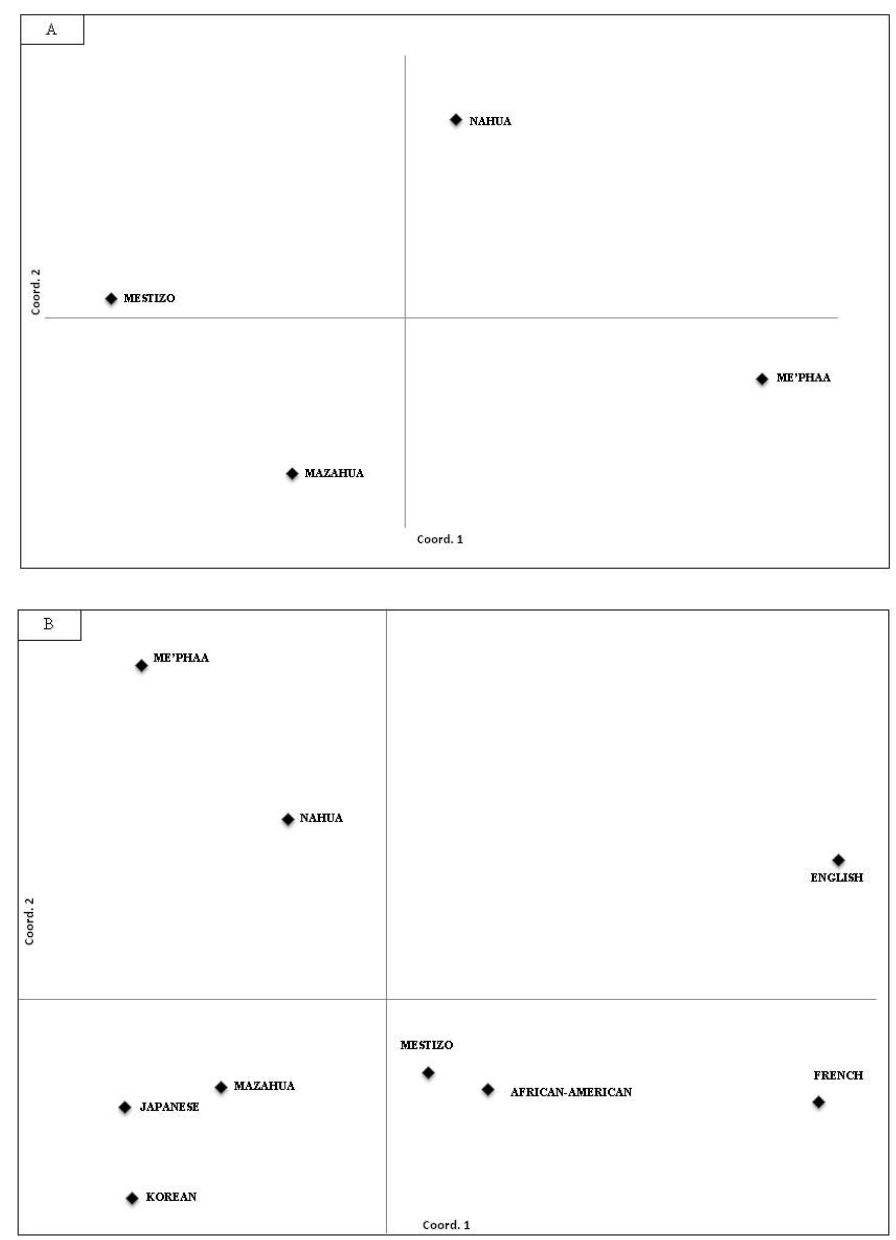

Figure 2. Principal components analysis. A. Genetic relationship of the $M M P-9$ microsatellite among Mexican Mestizo, Nahua, Mazahua, and Tlapaneco populations. B. Genetic relationship of $M M P-9$ among Mexican Mestizo, Nahua, Mazahua, Tlapaneco, Korean, Japanese, English, French, and African American populations. 


\section{Comparison with other populations}

To assess the genetic relationship with other populations, PCA was performed. We compared our data with those of Asian (Japanese and Korean populations), European (French and English populations), and African-American (USA) populations (Maeda et al., 2001; Zhang et al., 2001; Ferrand et al., 2002; Lamblin et al., 2002; Lee et al., 2010). The first component separated Mestizo, Mazahua, Asian, African-American, and French populations from the rest. Of note, the second component separated Amerindian and Asian populations from the other populations (Figure 2B). In addition, $F_{\mathrm{ST}}$ values were determined to evaluate the variation of allele frequencies among populations. Our results showed that the Mestizo population differed slightly from Mazahua (0.0257), Nahua (0.0410), Asian ( $\geq 0.0500$ : Japanese 0.0527 and Korean 0.0557), and African-American (0.0277) populations, whereas a moderate difference was observed with the European population (0.0899). Nevertheless, statistical differences were found in all populations $(\mathrm{P} \leq 0.001)$. On the other hand, the Nahuas showed moderate differences with the Mazahuas (0.0700), Me'Phaas (0.0618), Japanese (0.0757), Korean (0.0897), and African-American (0.0674) populations. The Mazahua population exhibited moderate difference with the Me'Phaas (0.0724) and African-Americans (0.0766), both with statistically significant differences $(\mathrm{P}<0.05)$. However, the Mazahuas showed only a small difference with the Japanese (0.0024) and Korean (0.0067) populations, with no statistical difference $(\mathrm{P} \geq 0.5)$.

\section{DISCUSSION}

Genetic association studies are powerful tools for the identification of biomarkers for complex diseases and the development of personalized therapies (Lunetta, 2008). Accordingly, a myriad of genetic studies have been published, many of which were focused on replicating findings reported in European populations (Carlson et al., 2013). Recent reports suggested that genetic polymorphism could affect the pharmacokinetics and pharmacodynamics of drugs. This variability has been related to ethnicity, which has been shown to play a critical role in the response to different therapeutic agents (Ortega and Meyers, 2014). In addition, robust evidence has pointed out that the prevalence and even the severity of different diseases differ among ethnic groups (Ortega and Meyers, 2014). Hence, it is crucial to evaluate the genetic architecture of polymorphic markers in populations with multi-ethnic backgrounds in an attempt to identify a genuine associations in these populations (Freedman et al., 2004).

In this study, we have shown the inter-ethnic variation of the $M M P-9$ microsatellite in the Mexican population. High genetic variability was observed $(k=13)$, even higher than that reported for African-American populations $(k=11)$ (Ferrand et al., 2002). This diversity was stronger in the Mestizo population, which exhibited the most frequent alleles of European $\left(\mathrm{CA}_{14}\right)$, Asian $\left(\mathrm{CA}_{21}\right)$, and African-American $\left(\mathrm{CA}_{>21}\right)$ populations, as well as significant heterogeneity (6\%, P $\leq$ 0.0001) (Ferrand et al., 2002; Fiotti et al., 2006; Lee et al., 2010). These findings were corroborated with PCA and genetic distance analyses, which showed relative proximity between the Mestizo population and their historical parental populations (Asian, European, and African) (Salazar-Flores et al., 2010). These characteristics were related to admixture of populations that showed dissimilar ethnic backgrounds and consequently differences in allelic distributions. However, the differences in the allele frequencies were also related to several demographic events such as bottlenecks, migrations, and rapid growth, which are known to modify genetic diversity as well as genetic structure (Pritchard et al., 2000). 
The Amerindian populations exhibited less diversity than the Mestizos; low diversity is characteristic of inbred and isolated populations such as the Me'Phaas, who settled in the uplands of Guerrero's Sierra (Bonilla et al., 2005). However, the Nahua population showed blatant diversity $(k=9)$. Nahuas, formerly Aztecs, are the most representative ethnic group of contemporary Mexico; these peoples have occupied the CVM since approximately 3000 years ago. In consequence, they have maintained genetic flow with the Mestizos (Vargas-Alarcon et al., 2007). In addition, this high diversity might also be related to the gene flow between the Nahuas and Mazatecos, an Amerindian group from Oaxaca, Veracruz, and Puebla that adjoins with Nahuas populations settled in Southeast Puebla, which could explain the differences found between the Nahuas and Mestizos $\left(F_{\mathrm{sT}}=0.0471, \mathrm{P} \leq 0.0001\right)$ (Castro-Sanchez and Escobedo-de la Pena, 1997; CDI, 2010). The Mazahua population showed a genetic relationship with the Mestizos $\left(F_{\mathrm{sT}}=0.02566, \mathrm{P}=0.6570\right)$. The Mazahuas inhabit the northwest region of the State of Mexico (CVM) and have maintained commercial and occupational interrelation with the Mestizos from Toluca (State of Mexico) and Mexico City (Cardoso-Saldana et al., 2006). Furthermore, this group also presented some similarities with Japanese $\left(F_{\mathrm{sT}}=0.0024\right.$, $\mathrm{P}=0.0641)$ and Korean $\left(F_{\mathrm{sT}}=0.0067, \mathrm{P}=0.0357\right)$ populations. These similarities could be related to a common ancestor ( 12,000 years ago) (Brown et al., 1998).

The study of the interethnic variation in the allele distribution of $M M P-9$ allowed the elucidation of specific features, possibly related to the history of the population that has exhibited such traits. Nevertheless, the genetic differences found in the populations reflect dissimilar frequency distributions as well as the action of natural selection (Wilson Sayres et al., 2014). Therefore, to clearly determine the amount of variation that occurs within the populations studied, it is necessary to use neutral loci such as mitochondrial DNA and the non-recombinant region of the Y-chromosome (Underhill and Kivisild, 2007). With this in mind, we had previously demonstrated a genetic substructure in the Mexican Mestizo population using a combined 15 DNA index system-STR (CODIS). This finding was corroborated using patrilineal markers (Salazar-Flores et al., 2010; Noris et al., 2012). As stated above, other demographic events can produce distinct levels of population structure, which could be related to mating practices, ancestral patterns, and even social history (Suarez-Kurtz and Pena, 2006; Ortega and Meyers, 2014). Thus, demography, genetics, and disease converge and add complexity to genetic association studies (Crawford, 2006). This complexity is even greater in inbred and recently appearing populations (10-15 generations) such as Latin-Americans, in which ancestry generates distinct levels of population substructure (Johnson et al., 2011; Noris et al., 2012). As a consequence, allele variability may only depict dissimilar ethnic backgrounds rather than an association of genes with diseases or therapeutic efficacy (Yasuda et al., 2008; Ortega and Meyers, 2014).

Our report provides compelling evidence for the crucial role that genetic diversity plays in association studies in complex populations. However, further research is needed in order to explain the severity and prevalence of various diseases in different ethnic groups, and to understand the impact of demographic events in phenotype-genotype associations. To our knowledge, this report constitutes the first examination of inter-ethnic variation of $M M P-9$ in the Mexican population.

In conclusion, our data provide evidence for the existence of inter-ethnic variation of $M M P-9\left(-90(\mathrm{CA})_{12-27}\right)$, which has shown a possible relationship to various diseases of cardiovascular or neurodegenerative nature, and cancer. Our data could be used as a powerful tool to avoid spurious associations in the search for genetic biomarkers. 


\section{ACKNOWLEDGMENTS}

Research partially supported by the Kellogg's Nutritional Institute Mexico (to R. Gómez). We thank the BIMODI Laboratory and coworkers for helping in the logistics of sample collection and processing as well as Unidos por la Montaña A.C., which supported the sampling of Me'Phaa population. We are grateful to all the Amerindian people for their enthusiastic participation; their collaboration made this study possible. We also thank Laboratorio de Genómica, Proteómica y Metabolómica from LaNSE-Cinvestav for helping with the genotyping processes as wells as Laboratorios de Especialidodes Inmunológicas S.A. de C.V., especially Laura Padierna Mota, Ph.D., for her contribution in some molecular reagents. Ultimately, we thank the Consejo Nacional de Ciencia y Tecnología (Conacyt) for scholarship \#347076 granted to Rafael Camacho Mejorado during his Ph.D.

\section{REFERENCE}

Belkhir KBP, Chikhi L, Raufaste N and Bonhomme F (1996). GENETIX 4.05, logiciel sous Windows TM pour la génétique des populations. Laboratoire Génome, Populations, Interactions. Montpellier (France), CNRS UMR 5171, Université de Montpellier II. Available at [http://kimura.univ-montp2.fr/genetix/].

Bonilla C, Gutierrez G, Parra EJ, Kline C, et al. (2005). Admixture analysis of a rural population of the State of Guerrero, Mexico. Am. J. Phys. Anthropol. 128: 861-869.

Brown MD, Hosseini SH, Torroni A, Bandelt HJ, et al. (1998). MtDNA haplogroup X: An ancient link between Europe/ Western Asia and North America? Am. J. Hum. Genet. 63: 1852-1861.

Cardoso-Saldana G, Pena-Diaz A, Zamora-Gonzalez J, Gomez-Ortega R, et al. (2006). Ethnicity and lipoprotein(a) polymorphism in Native Mexican populations. Ann. Hum. Biol. 33: 202-212.

Carlson CS, Matise TC, North KE, Haiman CA, et al. (2013). Generalization and dilution of association results from European GWAS in populations of non-European ancestry: the PAGE Study. PLoS Biol. 11: e1001661.

Castro-Sanchez H and Escobedo-de la Pena J (1997). Prevalence of non insulin dependent diabetes mellitus and associated risk factors in the Mazatec population of the State of Oaxaca, Mexico. Gac. Med. Mex. 133: 527-534.

CDI (Comisión Nacional para el Desarrollo de los Pueblos Indígenas) (2010). Los Pueblos Indígenas de México. Available at [http://www.cdi.gob.mx/index.php]. Accessed June 20, 2014.

Choudhry S, Coyle NE, Tang H, Salari K, et al. (2006). Population stratification confounds genetic association studies among Latinos. Hum. Genet. 118: 652-664.

Crawford MH (2006). Anthropological genetics: theory, methods and applications. 1st edn. Cambridge Universtiy Press, New York, 112.

Dabek J, Glogowska-Ligus J and Szadorska B (2013). Transcription activity of MMP-2 and MMP-9 metalloproteinase genes and their tissue inhibitor (TIMP-2) in acute coronary syndrome patients. J. Postgrad. Med. 59: 115-120.

Excoffier L, Laval G and Schneider S (2005). Arlequin (Version 3.0): an integrated software package for population genetics data analysis. Evol. Bioinform. Online 1: 47-50.

Ferrand PE, Parry S, Sammel M, Macones GA, et al. (2002). A Polymorphism in the matrix metalloproteinase-9 promoter is associated with increased risk of preterm premature rupture of membranes in African Americans. Mol. Hum. Reprod. 8: 494-501.

Fiotti N, Altamura N, Fisicaro M, Carraro N, et al. (2005). MMP-9 microsatellite polymorphism: association with the progression of intima-media thickening and constrictive remodeling of carotid atherosclerotic plaques. Atherosclerosis 182: 287-292.

Fiotti N, Altamura N, Fisicaro M, Carraro N, et al. (2006). MMP-9 microsatellite polymorphism and susceptibility to carotid arteries atherosclerosis. Arterioscler. Thromb. Vasc. Biol. 26: 1330-1336.

Flex A, Giovannini S, Biscetti F, Liperoti R, et al. (2013). Effect of proinflammatory gene polymorphisms on the risk of Alzheimer's disease. Neurodegener. Dis. 13: 230-236.

Fornoni A, Wang Y, Lenz O, Striker LJ, et al. (2002). Association of a decreased number of d(CA) repeats in the matrix metalloproteinase-9 promoter with glomerulosclerosis susceptibility in mice. J. Am. Soc. Nephrol. 13: 2068-2076.

Freedman ML, Reich D, Penney KL, McDonald GJ, et al. (2004). Assessing the impact of population stratification on genetic association studies. Nat. Genet. 36: 388-393. 
INEGI (2007). XII Censo general de población y vivienda 2000. Instituto Nacional de Estadística y Geografía, México. Available at [http://www.inegi.org.mx/est/contenidos/Proyectos/ccpv/cpv2000/].

Johnson NA, Coram MA, Shriver MD, Romieu I, et al. (2011). Ancestral components of admixed genomes in a Mexican cohort. PLoS Genet. 7: e1002410.

Juarez-Cedillo T, Zuniga J, Acuna-Alonzo V, Perez-Hernandez N, et al. (2008). Genetic admixture and diversity estimations in the Mexican Mestizo population from Mexico City using 15 STR polymorphic markers. Forensic Sci. Int. Genet. 2: e37-e39.

Kader AK, Shao L, Dinney CP, Schabath MB, et al. (2006). Matrix metalloproteinase polymorphisms and bladder cancer risk. Cancer Res. 66: 11644-11648.

Lacchini R, Metzger IF, Luizon M, Ishizawa M, et al. (2010). Interethnic differences in the distribution of matrix metalloproteinases genetic polymorphisms are consistent with interethnic differences in disease prevalence. DNA Cell. Biol. 29: 649-655.

Lamblin N, Bauters C, Hermant X, Lablanche JM, et al. (2002). Polymorphisms in the promoter regions of MMP-2, MMP-3, MMP-9 and MMP-12 genes as determinants of aneurysmal coronary artery disease. J. Am. Coll. Cardiol. 40: 43-48.

Lee YJ, Kang SW, Baek HJ, Choi HJ, et al. (2010). Association between matrix metalloproteinase 9 promoter polymorphisms and Behcet's disease. Hum. Immunol. 71: 717-722.

Lunetta KL (2008). Genetic association studies. Circulation 118: 96-101.

Maeda S, Haneda M, Guo B, Koya D, et al. (2001). Dinucleotide repeat polymorphism of matrix metalloproteinase-9 gene is associated with diabetic nephropathy. Kidney Int. 60: 1428-1434.

Mazzotti DR, Singulane CC, Ota VK, Rodrigues TP, et al. (2014). Association of APOE, GCPII and MMP9 polymorphisms with common diseases and lipid levels in an older adult/elderly cohort. Gene 535: 370-375.

Noris G, Santana C, Meraz-Rios MA, de Lourdes Munoz M, et al. (2012). Mexican Mestizo population sub-structure: Effects on genetic and forensic statistical parameters. Mol. Biol. Rep. 39: 10139-10156.

Ortega VE and Meyers DA (2014). Pharmacogenetics: implications of race and ethnicity on defining genetic profiles for personalized medicine. J. Allergy Clin. Immunol. 133: 16-26.

Pritchard JK, Stephens M and Donnelly P (2000). Inference of population structure using multilocus genotype data. Genetics 155: 945-959.

Salazar-Flores J, Dondiego-Aldape R, Rubi-Castellanos R, Anaya-Palafox M, et al. (2010). Population structure and paternal admixture landscape on present-day Mexican-Mestizos revealed by Y-STR haplotypes. Am. J. Hum. Biol. 22: 401-409.

Sawaya S, Bagshaw A, Buschiazzo E, Kumar P, et al. (2013). Microsatellite tandem repeats are abundant in human promoters and are associated with regulatory elements. PLoS One 8: e54710.

Shimajiri S, Arima N, Tanimoto A, Murata Y, et al. (1999). Shortened microsatellite d(CA)21 sequence down-regulates promoter activity of matrix metalloproteinase 9 gene. FEBS Lett. 455: 70-74.

Suarez-Kurtz G and Pena SD (2006). Pharmacogenomics in the Americas: the impact of genetic admixture. Curr. Drug Targets 7: 1649-1658.

Underhill PA and Kivisild T (2007). Use of y chromosome and mitochondrial DNA population structure in tracing human migrations. Annu. Rev. Genet. 41: 539-564.

Van den Steen PE, Dubois B, Nelissen I, Rudd PM, et al. (2002). Biochemistry and molecular biology of gelatinase B or matrix metalloproteinase-9 (MMP-9). Crit. Rev. Biochem. Mol. Biol. 37: 375-536.

Vargas-Alarcon G, Moscoso J, Martinez-Laso J, Rodriguez-Perez JM, et al. (2007). Origin of Mexican Nahuas (Aztecs) according to HLA genes and their relationships with worldwide populations. Mol. Immunol. 44: 747-755.

Wilson Sayres MA, Lohmueller KE and Nielsen R (2014). Natural selection reduced diversity on human y chromosomes. PLoS Genet. 10: e1004064.

Yasuda SU, Zhang L and Huang SM (2008). The role of ethnicity in variability in response to drugs: focus on clinical pharmacology studies. Clin. Pharmacol. Ther. 84: 417-423.

Zhang B, Dhillon S, Geary I, Howell WM, et al. (2001). Polymorphisms in matrix metalloproteinase-1, -3, -9, and -12 genes in relation to subarachnoid hemorrhage. Stroke 32: 2198-2202. 